\title{
An Empirical Study on the Precise Employment Situation-Oriented Analysis of Digital-Driven Talents with Big Data Analysis
}

\author{
Lin Li ${ }^{1}{ }^{1}$ and Sang-Bing Tsai $\mathbb{D}^{2}$ \\ ${ }^{1}$ Youth League Committee, Xi'an University of Science and Technology, Xi'an 710054, China \\ ${ }^{2}$ Regional Green Economy Development Research Center, School of Business, Wuyi University, Nanping, China
}

Correspondence should be addressed to Lin Li; zclwkd@126.com

Received 12 November 2021; Revised 13 December 2021; Accepted 18 December 2021; Published 4 January 2022

Academic Editor: Gengxin Sun

Copyright (C) 2022 Lin Li and Sang-Bing Tsai. This is an open access article distributed under the Creative Commons Attribution License, which permits unrestricted use, distribution, and reproduction in any medium, provided the original work is properly cited.

\begin{abstract}
This paper conducts an in-depth research analysis on the precise employment of college graduates in the context of big data using a number-driven approach. The textual information of the study is obtained by using in-depth interviews, and the evaluation index system of college students' employment quality is constructed by combining the step-by-step coding method with rooting theory. The research on the current situation of employment recommendation platform research and the application status of big data in the employment recommendation platform is explored by using a bibliometric approach. And the innovative use of web crawler technology is used to comprehensively understand the recommendation function and status quo of the same type of recommendation platform, which provides a reference for the research of this platform. Based on the preliminary analysis of platform requirements and overall design, the overall design and functional implementation of the big data employment recommendation platform are carried out by using big data crawler technology, big data architecture technology, text mining technology, database technology, etc. The construction of a recommendation module based on user history information, a recommendation based on real-time user online behavior data, and hybrid recommendation carried out on the recommendation module to grasp all-round the platform is built based on a stakeholder perspective. Based on the platform construction, the initial platform operation and maintenance management mechanism was established from the stakeholder's perspective. The Pearson correlation coefficient is used to objectively evaluate the current situation of talent supply in universities and talent demand in enterprises from the perspective of image and data. In the research on the development status of the big data education industry, the Lorenz curve and Gini coefficient are used to match the status of new big data majors with their college construction volume in each province and provide data support for the reasonable adjustment of majors setting in each province according to the education level.
\end{abstract}

\section{Introduction}

One of the evaluation criteria for the success of higher education is the employment situation of students, in which the employment information management of students is one of the important work contents in the employment process. With the arrival of the era of big data, the information construction of college students' employment work faces new requirements [1]. It is extremely important to strengthen the information construction of employment services by effectively using network technology, explore a new road of employment service information suitable for its development, and help students to be employed faster, better, and more harmoniously. And as there is increased employment recommendation research to focus on user behavior data analysis and text mining, the use of big data technology to deal with the employment recommendation problem has received increased attention in both theoretical research and practical application. Recommendation platforms based on recommendation models are the most effective personalization techniques in the era of big data. However, conventional recommendation means tend to 
simply analyze and model related to users' online behavior, while ignoring the development of student users' attribute characteristics. Most of the activities performed by student users on the Internet are similar, and it is obvious that the way to classify students' attributes and build recommendation models by simple user's online behavior does not achieve the expected effect [2]. With the widespread use of social networks in recommendation, and the continuous research on students' school data, how to effectively use these unstructured data as the constituent attributes of userprofiles in recommendation models will be the key and difficult point to promote the development of personalization technology in the era of big data.

In terms of theory, it combines stakeholder theory and platform-related theory and fully explores the big data-related theory, while using bibliometric research methods to review the big data employment recommendation platform and big data employment recommendation algorithm, to comprehensively understand the development status and development trend of employment recommendation platform and employment recommendation algorithm in the context of big data. Big data not only constructs the platform construction technically, but also takes the management strategies such as big data opening and related theories as guidance and fully applies them to the platform operation and maintenance management, which realizes the effective combination of theoretical research and practice [3]. This provides effective theoretical support for the big data employment recommendation platform and employment recommendation algorithm as well as the operation and maintenance management aspect of the big data employment platform. In practice, the research on the construction of employment recommendation platforms and employment information recommendation combined with big data technology is a relatively new research direction, and the employment recommendation platform will be landed under the guidance of theoretical research, and the big data employment recommendation platform in a good operation state will be a new direction of employment recommendation platform research in the era of big data, and it will be a reference for the traditional recruitment industry on how to effectively use the Internet and link big data to better improve employment [4]. It will serve as a reference and guidance for the traditional recruitment industry on how to effectively use the Internet and link big data to better improve the quality and efficiency of employment recommendation. With the popularization of the Internet, increased enterprises are releasing their recruitment information through the medium of recruitment network platform, and the emergence of network recruitment form can effectively solve the problem of the limitations of the existing data statistics, which is also the information channel that can best reflect the market demand for talents; the information is concentrated on the web, with a large amount of data [5].

How to effectively use network technology to strengthen the construction of employment service informatization, explore a new path of employment service informatization suitable for their own development, and help students get faster, better, and more harmonious employment is of extremely important significance. The first is to construct an employment quality evaluation model, which helps college students improve their employment quality by their self-test. This paper obtains textual information through in-depth interviews, combines it with rooting theory to screen indicators of employment quality influencing factors, and, on this basis, constructs an employment quality evaluation model of college students in the new economic background, which can evaluate the employment quality of college students more comprehensively by studying the factors influencing the employment quality of college students from different research perspectives. Secondly, it is to provide a reference for teaching in colleges and universities and provide a reference basis for government policies. By constructing the evaluation model of college students' employment quality in the context of the new economy and conducting empirical analysis, it is more targeted to analyze the level of college students' employment quality in Jinan City and the influence indexes, which can provide a reference for the teaching content and curriculum of colleges and universities and provide the basis for government policies.

\section{Current Status of Research}

With the employment of college students changing from the state allocation in the planned economy to the two-way selection in the employment market, the employment situation of college students was once increasingly severe, and employment guidance services have been established to effectively help college students in employment, while information technology and network technology, to promote employment, play an important role in the employment guidance services [6]. Therefore, many scholars began to actively focus on and study the problem of information construction of employment work [7]. It is proposed that colleges and universities should provide information platforms, fully integrate information resources, establish employment information networks connecting various regions, institutions, and industries, and realize information sharing, to effectively establish information communication channels among colleges and universities, employers, and graduates [8]. At the same time, related literature also points out that there are problems in the current informatization construction of employment services, proposing that the construction of employment informatization in colleges and universities lacks overall system planning and the lack of linkage mechanism among colleges and universities, resulting in poor channels of employment information dissemination [9]. It is proposed that the employment informatization construction of colleges and universities is still irregular and out of place, which leads to the lack of good information support and helps graduates in their job search. The necessity of colleges and universities to strengthen the requirements of information construction of college students' employment services and integrate and share employment information resources is proposed [10].

Because of the current situation faced by the employment information construction of college students, related scholars have studied the application of big data in college 
employment, which can not only improve the employment rate of schools but also help graduates find better employment by collecting students' job-seeking intention, professional assessment, personality analysis, career evaluation, and other information, and comprehensively analyzing and correspondingly matching them with the scattered, complex, and huge recruitment information in the social environment [11]. It is considered that, with the continuous improvement of the level of information construction of higher education, universities have accumulated certain data information in the management process and should better serve the employment of graduates through information technology, and these studies provide support for strengthening the information construction of college students' employment services [12]. After the theoretical exploration of algorithm based on the framework of big data technology [13], the mature research and application practice of big data in technology and the full combination with algorithms have enhanced the possibility and efficiency of big data in solving real-world problems. And in the context of research on big data technology and applications, it is feasible and efficient to study graduate employment recommendation platforms with the perspective of big data technology as a background and technical support.

Collecting the theory, the history and development of stakeholder theory are described in detail, further classifying the stakeholders according to different dimensions as primary stakeholders, secondary stakeholders, intended stakeholders, and potential stakeholders. And Mitchell's scoring method was developed to formulate stakeholders. Mitchell's scoring method emphasizes three attributes of stakeholders, namely, urgency, power, and legitimacy. Urgency refers to the ability of a group to immediately attract the attention of managers, and it emphasizes the degree to which a group is valued by the platform; power refers to the ability of a group to influence the decision making ability of managers, and it emphasizes the impact of a group's behavior on the platform thereby causing decision-makers to make decisions about the direction of the platform, and legitimacy refers to the group being given a specific claim on the firm's resources, and it emphasizes the ability of a group to take up resources in the platform.

\section{Analysis of the Precise Employment Situation of Digitally-Driven College Graduates}

3.1. Big Data Digitally Driven Algorithm Design. The data is crawled according to certain rules for web pages with timed and untimed data. Considering the timeliness and a large amount of data, this study selects the data of full-time section and internship section in the campus recruitment section of WiseLinks Recruitment and MileagePlus, takes software engineering as an example to crawl the data of enterprises' requirements for software engineering jobs on the platforms of WiseLinks Recruitment and MileagePlus, and conducts relevant text analysis to guide the extraction of keywords of information related to graduates and the matching of recommendation algorithms at a later stage [14]. Overall, in terms of recommendation system, the recommendations of popular search, popular industry, popular enterprise, and popular position are more used in the recommendation section of each job search platform, which also reflects that the core of both college employment platform and general employment platform for job recommendation is focused on the recommendation of industry, enterprise, and position [15]. These three aspects of employment recommendations represent the basic needs of today's employment class platform recommendation system. At the same time, they have different recommendation preferences in three aspects: popular online application, popular internship, and popular presentation. From the platform side, there is a big difference in the operation and solution of recommendation systems between different platforms, and the homogeneity of the operation of the recommendation system is obvious in the same type of platform.

In the missing value processing after the data deduplication process, common data duplication generally includes two kinds, one for the record duplication (that is, there are several records about a certain or some characteristics of the value, which are the same), and the other for the characteristics of duplication (that is, there is a certain or some characteristics of the value, which are the same, but the name of the data features is different). Due to crawler technology reasons, or because the site itself may produce duplicate records, we take the above duplicate records to delete duplicate records to retain only one operation; this data duplication problem only includes the record duplicate type; we traverse the data; the call can complete the data deduplication. Because there is no association between the data, so the data cleaning only after the table misalignment check, due to the absence of information part of the subsequent data forward to deal with the misalignment of the table part of the phenomenon, first determines whether the missing information is the position; if the position information is complete, it is not processed, and vice versa, to delete the data column; this can end the data preprocessing operations, finishing the month as the time unit of the enterprise demand base data table, as shown in Table 1.

For the massive amount of data collected, we cannot store and use all of it. Take the one-card information as an example; it contains the usage information of students and staff, and for the usage of students, it contains the usage information of undergraduate and graduate students for different behavior patterns. In this paper, we mainly focus on the study of school students, so we need to first filter the data in combination with people, to eliminate the information of irrelevant people [16]. The data collected from university students also contains data anomalies due to uncertainties such as suspensions, retakes, and missed exams. In all cases, we use methods such as filtering to target groups and eliminate missing, abnormal, and redundant data. Ignore the development of the characteristics of student users' own attributes. Most of the activities performed by student users on the Internet are similar. It is obvious that the method of categorizing students' attributes and establishing a recommendation model through simple user network behaviors does not achieve the expected results. 
TABLE 1: Agreed employment.

\begin{tabular}{lcc}
\hline Attribute name & Type & Value \\
\hline XH & Int & 3 \\
XYCJSJ & Int & 6 \\
HTQX & Int & 8 \\
DWZGBM & Int & 113 \\
\hline
\end{tabular}

$$
Y_{t}=(1-p l) U_{t}, \quad t=1,2, \ldots, n .
$$

The model order is determined by creating autocorrelation and partial autocorrelation plots from the identified features, defining the phenomenon that the function plot tends to zero after a certain order as a truncated tail, and it is not possible to determine its range. The AIC or BIC criterion can be used to estimate possible or even seasonal parameter values, and thus to evaluate the model in order and select the optimal model.

$$
r_{k}=\frac{\sum_{t=k}^{m}\left(x_{t}+\Delta x\right)\left(x_{t-k}-\Delta x\right)}{\sum_{t=k}^{m}\left(x_{t-k}-\Delta x\right)^{2}} .
$$

In addition to the $r$-value, the $p$-value is also an important indicator that we must consider. It is meaningless to discuss whether two variables are correlated or not. It is meaningless to talk about the magnitude of the correlation coefficient without talking about the $r$-value, and the correlation between the two may be caused only by chance, so we must judge the significance level of the correlation between the two variables. $p$-value means the probability that there is no correlation between the variables, so the correlation analysis we perform is meaningful when the $p$-value is small enough. We generally set the threshold of significance level at $5 \%$, i.e., 0.05 . So, when the $p$-value is much greater than 0.05 , we cannot say that there is a significant correlation between the two variables even if the correlation coefficient is large; and we generally must talk about the magnitude of the correlation coefficient before the $p$-value meets the requirement.

$$
P\left(M_{i} \mid y_{1}, \ldots, y_{n}\right)=\frac{P\left(y_{1}, \ldots, y_{n} \mid M_{i}\right) P\left(M_{i}^{2}\right)}{P\left(y_{1}, \ldots, y_{n}\right)} .
$$

To construct the evaluation index system of college students' employment quality, it is necessary to consider various factors affecting employment quality as comprehensively as possible, and to establish the evaluation index system comprehensively and systematically, which helps reflect the current situation of college students' employment quality more truly and effectively. If the selected indicator system does not have operability, the evaluation objectives cannot be achieved. Therefore, when constructing evaluation indicators, the selection of indicators should not only consider the difficulty of obtaining indicators, but also consider whether the indicators can be quantified and the reliability of the indicators, as shown in Figure 1.

Fully used in the platform operation and maintenance management, to achieve an effective combination of theoretical research and practice, this provides effective theoretical support for the big data employment recommendation platform and employment recommendation algorithm, as well as the operation and maintenance management of the big data employment platform. The goal of user profiling is to describe the user object, fully recognize and understand the user needs, and achieve personalized recommendations. The ways to achieve user portraits are generally divided into formal means and nonformal means, with formal means mainly using text, voice, and image presentations, and nonformal means mainly using descriptions. And the common way to implement user portraits is like pixels in imaging technology to portray the characteristics of people, which is the label in portraits. The prerequisite for user portrait modeling is the labeling of user features, and the creation of user feature labeling is also the focus of the later section. User profiling involves structured and unstructured approaches to data, i.e., quantitative and qualitative user profiling $[17,18]$. The establishment of the tagging system in the qualitative user portrait mainly relies on manual customization at this stage, and the basis of customization is the common sense and consensus knowledge system. The verification involves the feedback process of users, and the accuracy of the user profile needs to be evaluated and fed back by the users.

$$
\begin{aligned}
\varphi_{1}(B) \nabla^{1} \nabla_{12}^{1} z_{t} & =\beta_{1}(B) a\left(B 2_{2}^{12}\right) \alpha_{t}, \\
z_{t} & =\varphi_{1} z_{1}-\varphi_{2} z_{2}+\varphi_{3} z_{3} \ldots
\end{aligned}
$$

Under the active promotion of employment management departments at all levels, colleges and universities have explored and established their employment management service systems, and through continuous exploration and indepth practice of employment work informatization construction, certain results have been achieved. From the establishment of employment service network to now, a large amount of information has been accumulated, while various social talent recruitment websites have also started to pay attention to the employment market of college graduates and set up campus recruitment network channels specifically for graduates, effectively promoting the development of graduate employment work. The information construction has improved the overall process and working methods of college students' employment work, enhanced the efficiency and quality of college employment management services, and provided higher quality and standardized services for college students and employers, which has a positive effect on college students' employment, as shown in Figure 2. To further explore whether high-frequency words can play a dominant role in the text, this study introduces the method of keyword cooccurrence; that is, the relevant keywords appear in two different employment demand species; then, it is determined that the two keywords appear more often at the same time, and the degree of association is higher, so that it can be further determined which high-frequency keywords have higher centrality and more obvious importance. See Figure 2.

In this study, high-frequency words with frequencies greater than or equal to 1000 were selected and imported into the word frequency analysis tool BibExcel for cooccurrence calculation of high-frequency words, and the 


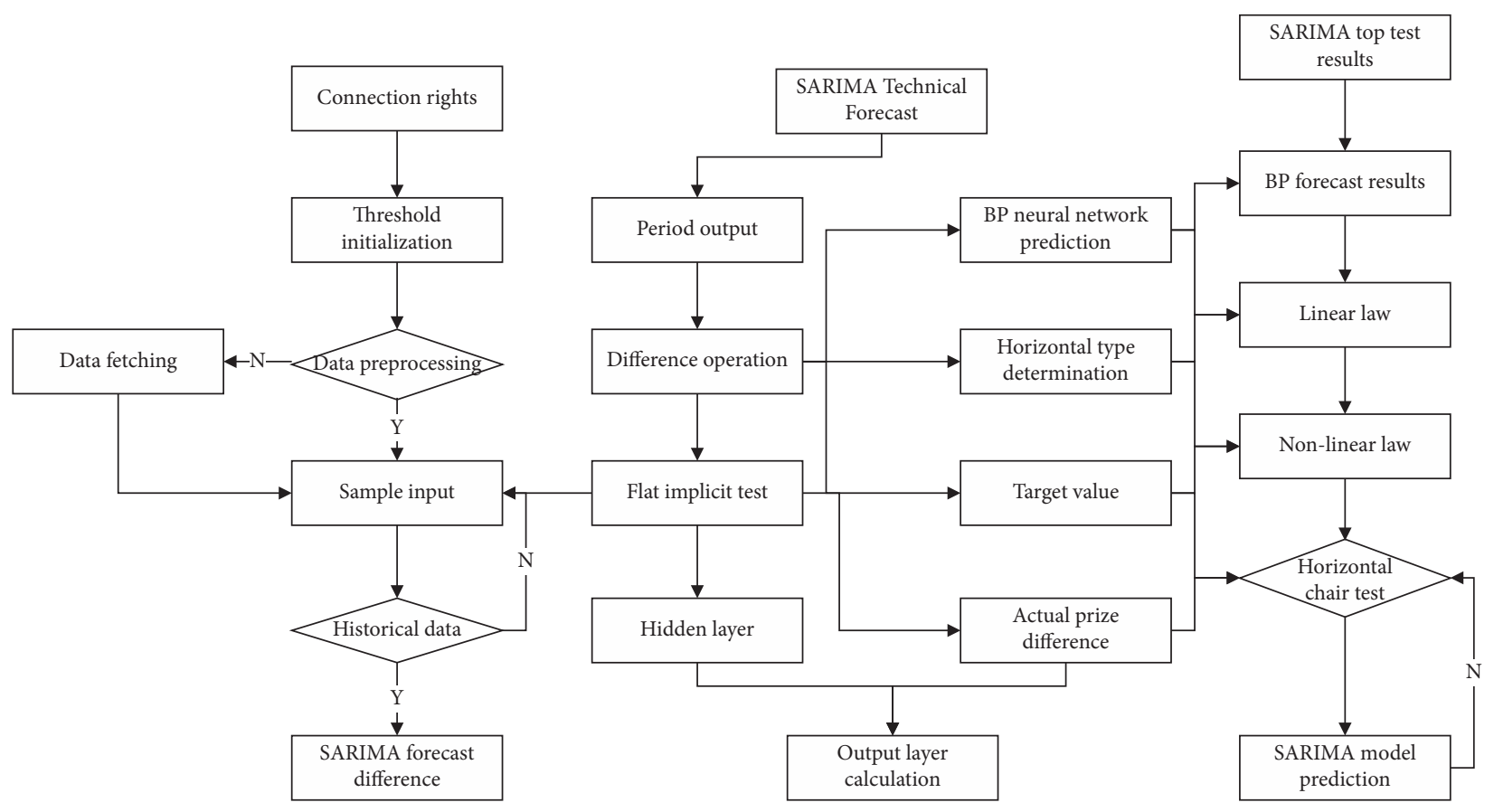

Figure 1: Flowchart for modeling a big data portfolio model.

cooccurrence matrix of high-frequency keywords with cooccurrence frequencies $\geq 2$ was imported into the social network analysis software UCINET for centrality analysis of high-frequency keywords, and the results were visualized with the visualization software NetDraw for visual presentation of keyword cooccurrence.

\subsection{Analysis of the Precise Employment Situation of High} School Graduates. Big data technology can analyze a large amount of data such as current economic development, employment information of previous students, recruitment information of enterprises, and the flow of graduates and predict the employment situation of various industries, salary level, competition situation of various regions, etc. Government employment guidance departments at all levels can arrange employment work in a coordinated manner; enterprises can carry out recruitment in a planned manner, provide reasonable salary packages, and find suitable job seekers; thus saving universities can carry out corresponding employment guidance in a planned and systematic manner to provide graduates with better employment guidance services; university graduates can establish a suitable employment outlook according to their majors, combine their interests, strengths, and personal development intentions, clarify job-seeking goals, and make adequate preparation for job applications, thus strengthening the relevance of employment work carried out by all parties $[19,20]$. Through big data technology, when job seekers search for career information, they can show relevant career information by setting information keywords and automatically block irrelevant information to improve the efficiency of information acquisition. Through the comprehensive analysis of big data technology, some false recruitment information on the network can be quickly identified and eliminated, and the information audit function is strengthened to reduce the probability of job seekers being cheated [20,21]. University graduates can establish a suitable employment outlook based on their own majors, combined with their own interests, expertise, and personal development intentions, clarify their job search goals, and make adequate preparations for employment, to strengthen the pertinence of all parties in the employment work.

The recommendations of people who want to know are divided into two cases; one of them is collaborative filtering based on tags, and the other one is the similarity of an online assessment. The algorithm is an algorithm that automatically generates multiple-choice questions for the system users to take knowledge quizzes based on the questions that already exist in the database. Since this system designates the test questions as multiple choice questions and in the test paper, each question has a unique ID to correspond to a score, so after the system user submits the test paper, the system backend automatically compares their chosen answer with the correct answer precisely, the ID corresponding to the answer, and then calculates the score for that system user as shown in Figure 3.

The machine learning part makes full use of the real-time data parallelization processing capabilities in Mahout and the corresponding algorithms for data modeling, mainly including user-based collaborative filtering and item-based collaborative filtering. It is also possible to customize algorithms, such as content-based and tag-based recommendations, to increase the effectiveness of modeling incremental data. For the construction of popular industries, jobs, enterprises, and other information boards mainly from the data crawlers of various Cosmos-type websites and the accumulation of data on this platform, this part of the data 


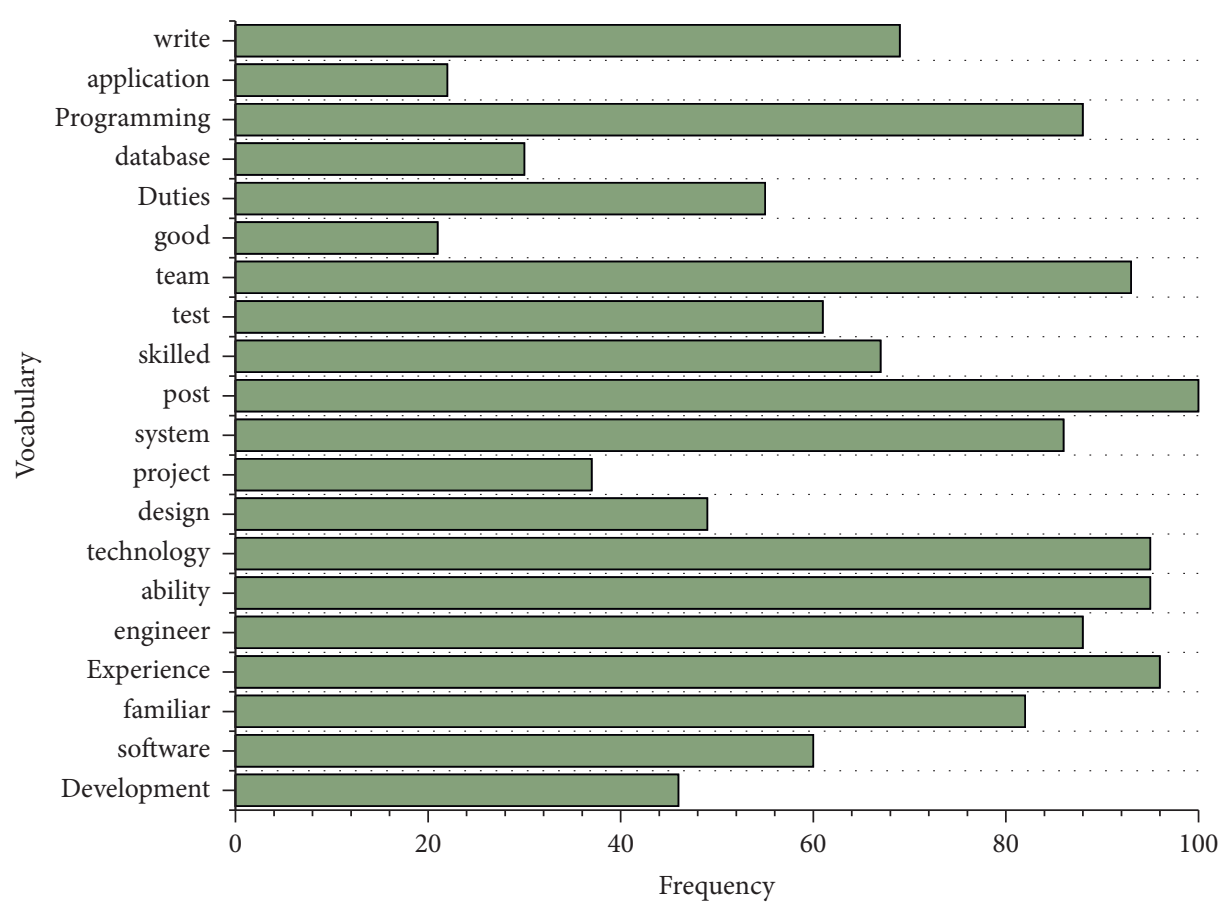

Figure 2: Distribution of high-frequency words.

display can be in the form of reports, but it can also be displayed with the effect of online dynamic graphics such as Charts.

In this paper, we mainly use the grid tuning method to adjust the hyperparameters of this model. It is specifically divided into coarse tuning and fine-tuning, and the difference between the two lies in the different granularity of tuning. In hyperparameter tuning, first, coarse tuning is performed; i.e., for each parameter, relatively large step size is used for the parameter to perform a grid search over a relatively large range of parameters. This has the advantage that we can spend a relatively short time to roughly estimate the best value for each hyperparameter. Secondly, we perform a "carpet" search for each hyperparameter using the finest granularity step, based on the best hyperparameter value obtained from the coarse tuning. In this way, the model will have better accuracy. See Figure 3.

Prediction is an important function of big data. Through the analysis of employment data of previous graduates, the corresponding employment trend can be predicted, which enables college students to grasp the employment situation early and adjust the employment mentality in time; it enables colleges and universities to carry out targeted employment guidance work early and prepare for the employment of graduates with corresponding services. The emergence of online recruitment forms can effectively solve the limitations of existing data statistics, and this is also the information channel that best reflects the market's demand for talents; information is concentrated on the web, which has the characteristic of large data volume, relying solely on search. It is difficult for the engine to accurately obtain effective intelligence. The prediction of employment-related trends can be made through the relevant policies provided by the government employment management departments to understand the corresponding national employment policies; the state of the labor market and occupational demand in various regions can be understood through the national employment policies; the latest developments in the employment market can be understood through the media such as the Internet, newspapers, and magazines, and selfmedia, and specific information on occupations, and the occupational information in these media into a certain extent; it reflects the needs of enterprises and has an important guiding role in graduates' job search. Then, these data are analyzed and organized to predict employmentrelated trends, such as predicting the supply and demand of the relevant industries for the talents cultivated in their specialties and the demand trend for talents in their regions, to help graduates change their employment direction in time to achieve more reasonable employment and help adjust the talent training program in time according to the market demand, as shown in Figure 4.

Make full use of the employment information platform to build a platform for the exchange of employment information among graduates, employers, and schools, to realize the sharing and effective allocation of information resources. To improve the accurate information pushing mechanism at the school and faculty levels, relevant departments and faculties of the school divide the tasks and implement them at all levels, understand the employment status and willingness of each graduate through the employment counselor of the graduation class, and record in detail the employment intention of graduates in terms of geographic area, willingness, salary, and so on. At the same time, the school accurately grasps the nature of the employer, job content, recruitment conditions, and other 


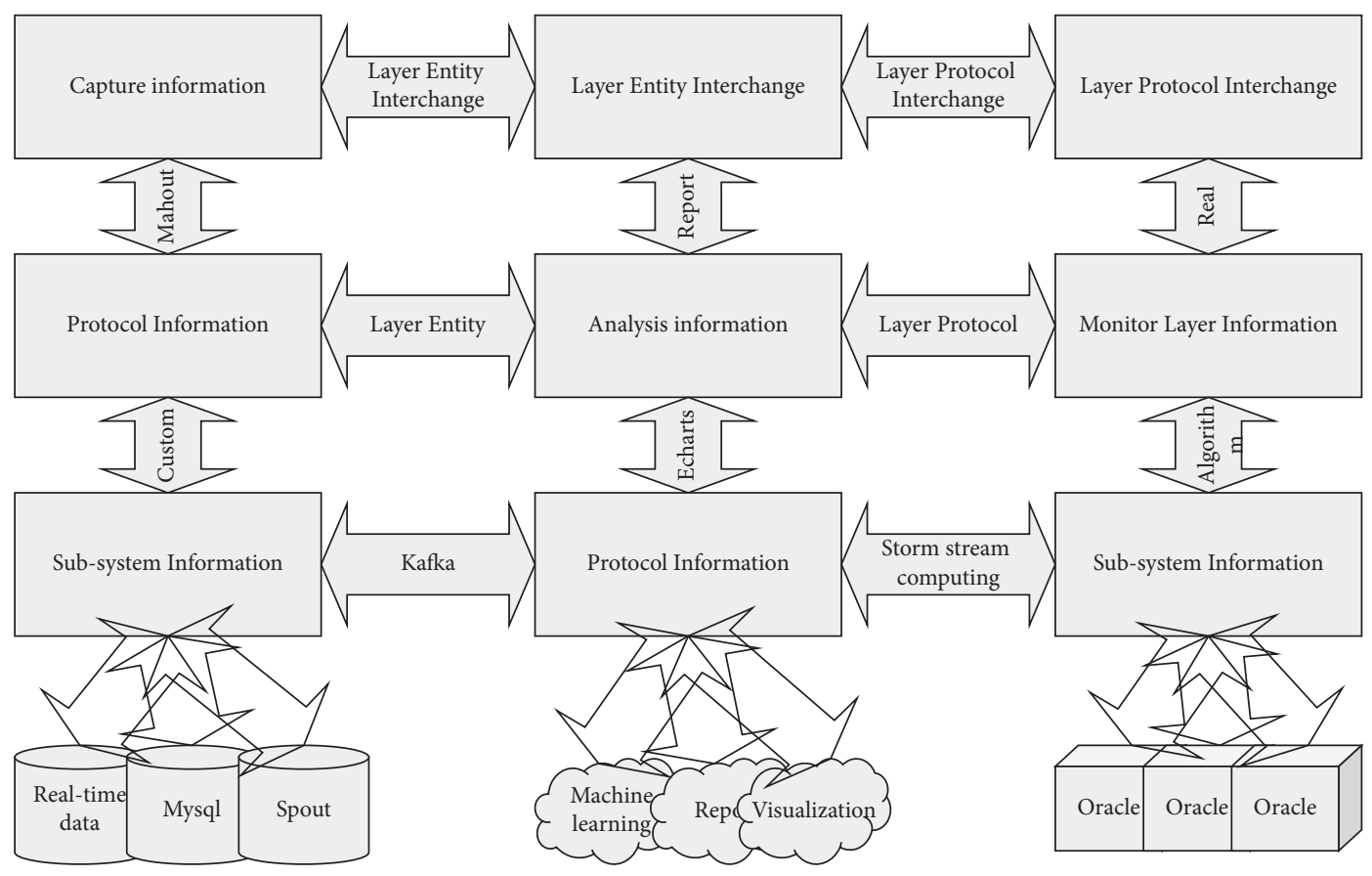

Figure 3: Behavioral recommendation architecture.

recruitment information, based on the database of graduates and the database of job demand information of the employer to do a good job of matching, intelligent matching of education, professional, geographical, and other keywords, for graduates and employers to accurately push the supply and demand information that meets the requirements. The research is mainly aimed at college students, so it is necessary to filter the data with personnel first, to eliminate the information of irrelevant personnel. As for the collected data of college students, it also includes phenomena such as abnormal data caused by uncertain factors such as suspension, retake, and missed exams. With the development of big data technology, the information construction also shows the momentum of diversification and innovation, and various emerging software has powerful service functions, so that graduates can receive employment information in time and participate in the whole process of carrying out employment work in school and communicate and discuss. The development of big data technology has also enriched the dissemination of information, the dissemination of information does not just rely on computers, smartphones, portable tablets, and smart wearable devices that have become the medium of information dissemination, and information platforms are no longer limited to the Internet sites real-time communication tools, and a variety of APP applications have become the new information platform, more convenient and fast dissemination of information; with the help of these platforms, job seekers can browse information, submit resumes, ask questions, and communicate at any time and anywhere, and corporate human resources departments can release information, screen resumes, answer questions, and video interviews at any time, greatly improving the convenience and success rate of job search.

\section{Analysis of Results}

4.1. Big Data Numerically Driven Algorithm Performance. As an example, the first half-semester employment slope of students characterizes the change in the frequency of employment during the first half of the semester. From Figure 5, it is known that the slope of employment in the first half of the semester is negatively associated with the employment of students. The frequency of employment reflects the physical health of the students, and the mean value of the employment slope is $(0.83,0.21,-0.46)$ for the three categories of students who are not employed, employed, and promoted, respectively. That is, the students in the failed employment category had the greatest rate of increase in employment during the first half of the semester, reflecting a declining trend in physical conditions for this group of students. The interval of the slope of the first half of the medical treatment for the three types of students is because the first half of the semester is the best time to prepare for exams and find a job, during which the physical health is particularly important, and poor physical health can lead to eventual employment failure. With this, we can determine the risk of a student's employment failure. See Figure 5.

The accuracy of the RF prediction model using only traditional features is 0.608 , and the accuracy of the LSTM prediction model using only temporal features is 0.571 . The model in this paper is constructed by fusing traditional features based on statistical analysis and temporal behavioral features based on deep learning, and its prediction accuracy reaches 0.708 , which is much higher than the above two. Therefore, it is realistic and feasible to predict students' employment through their school behavior data. Some colleges and universities focus on employment rate but ignore employment safety education, and the employment 


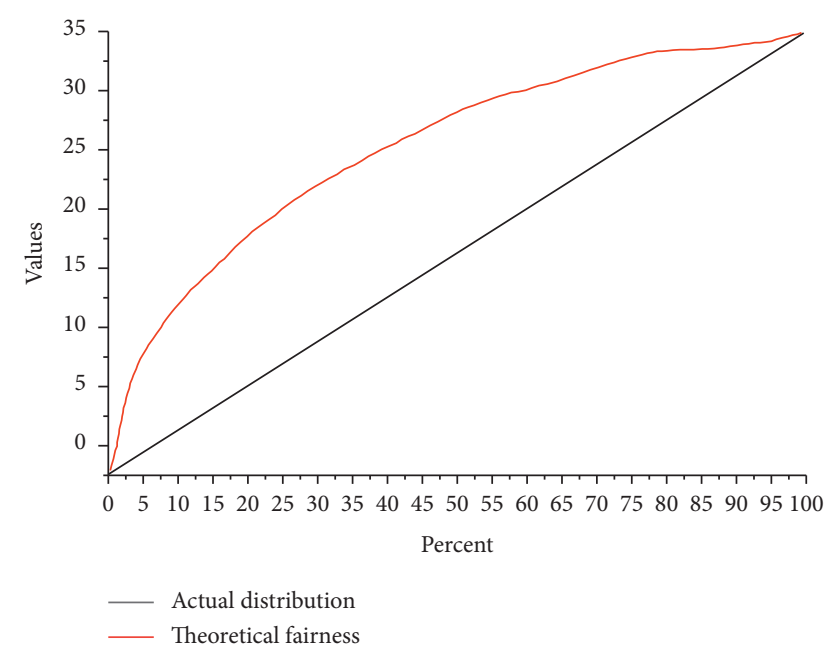

FIGURE 4: Lorenz curve matching situation.

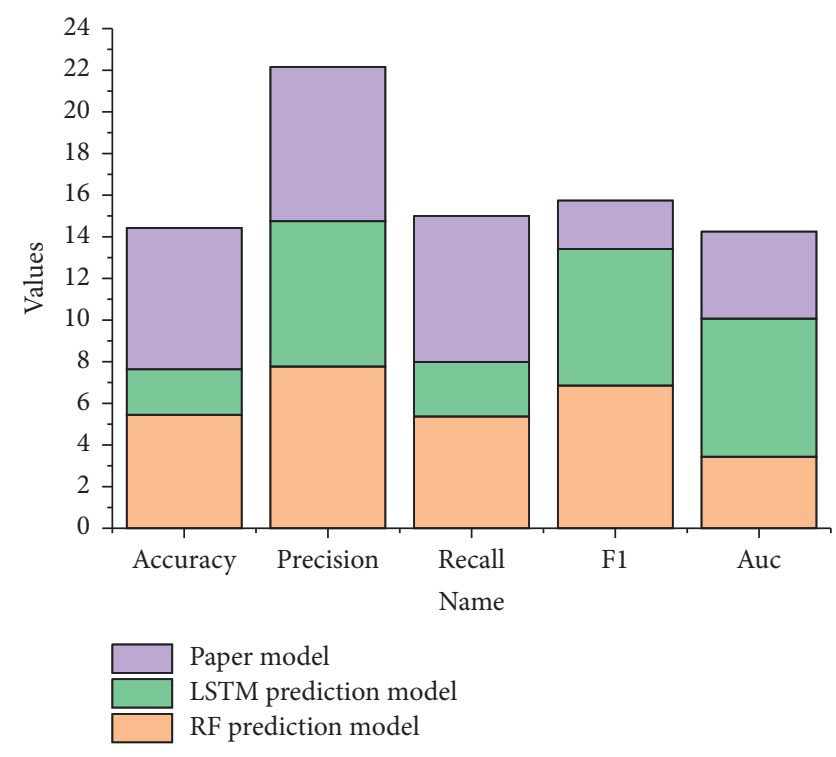

Figure 5: Cross-validation comparison results.

safety education for college students stays at the theoretical level, only explaining to college students the matters they should pay attention to when signing employment contracts, and less explaining the process link of online recruitment, the precautions they should take, how college students can protect their rights and interests, and how to protect their rights and interests when they are violated, and other problems college students may encounter. Insufficient teachers for employment guidance in colleges and universities: some colleges and universities do not have special teachers of employment guidance class, and the employment guidance class is taught by counselors; in turn, these teachers have more student management affairs, and they do not have comprehensive knowledge about employment guidance, and colleges and universities also seldom organize employment guidance teachers for relevant training and education, and college students lack professional employment concept education, which affects college students' full employment. On this basis, the employment quality evaluation model of college students under the background of the new economy is constructed, and the factors affecting the employment quality of college students are studied from different research perspectives, which can more comprehensively evaluate the employment quality of college students.

Figure 6 compares the differences in the scores of evaluation indicators of the quality of employment of university students by gender. The scores of each evaluation indicator are the average of the self-measurement scores of the respondents. In Figure 6, the mean values of labor compensation, housing fund, occupational welfare, and work pressure are relatively low, among which the mean value of male students is higher; the mean values of employment environment, social insurance, and employee relations of male and female university students are relatively high. In the remaining indicators, the mean values of male students are slightly higher or equal to those of female students. See Figure 6.

The fuzzy comprehensive evaluation method is a comprehensive evaluation method that transforms qualitative evaluation into quantitative evaluation, using fuzzy mathematical affiliation theory to evaluate things influenced by multiple factors. The basic idea is to determine the set of factors and the evaluation set of the evaluated object; to determine the weights of each factor and their affiliation vectors to obtain the fuzzy evaluation matrix; to carry out fuzzy operations and normalization according to the obtained fuzzy evaluation matrix and the weight vectors of each factor to obtain the fuzzy comprehensive evaluation results. The same type of platform has obvious homogeneity in the operation of the recommendation system. Traditional recruitment platforms have entered the recommendation field well, and their recommendation operations in industries, companies, internships, and presentations are in good condition, and they mainly adopt separate recommendation and display solutions for industries and companies.

It can be found that the self-measurement scores of job characteristics, compensation and benefits, career development, labor-management relations, employee recognition, and employment flexibility indicators at the normative level in the new economy are higher than the self-measurement values of college students in the traditional economy. Under the new economy, the highest score of the criterion-level indicators is job characteristics, and the lowest score is the indicator of employment flexibility, which verifies that the jobs under the new economy better meet the needs of college students in terms of work, and better meet the requirements of college students in terms of safety and security employment environment; the lowest score of the indicator of employment flexibility and the rapid development of the new economy provide more employment opportunities for college students at the same time but also place higher demands on college students. The new economy is more flexible in terms of employment, but under the current economic situation, more college students are forced to work under the pressure of livelihood 


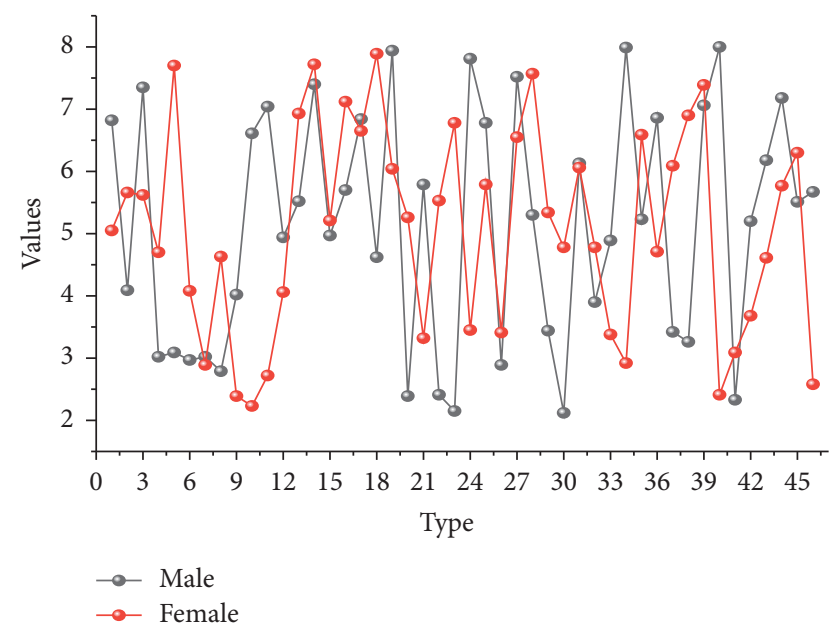

Figure 6: Comparison of indicators for evaluating the quality of employment of university students by gender.

and do not have better work freedom and work intensity, in line with the current employment situation of college students.

4.2. Results of Precise Employment Analysis of High School Graduates. In this study, the factors affecting the employment of master's degree in agricultural information technology are social factors, school factors, and personal factors through factor analysis of the questionnaire, followed by quantitative analysis of these three factors using hierarchical analysis to obtain the relative importance ranking of each factor affecting employment. The hierarchical analysis method requires the construction of a judgment matrix that meets the consistency requirements, and when the consistency ratio $\mathrm{CR}<0.1$, the judgment matrix passes the consistency test and can continue to analyze the weights of each index relative to the total index to evaluate the degree of importance and provide a reference for the training and employment of information technology graduate students through the improvement of the influencing factors. From the arrangement of the weight data, the course content has a crucial influence on employment, and with the development of science and technology, agricultural informatization education in agricultural and forestry colleges and universities in the context of big data should conform to the needs of the times and adjust the training mode with the actual needs of the country and society, so that the professional courses have high efficiency and practicality. From the cultivation programs of agricultural information technology in the seven agricultural and forestry colleges and universities investigated, we can see that there are certain limitations in the teaching structure of agricultural and forestry colleges and universities; compared with China's requirements for agricultural high-tech talents, the cultivation programs of agricultural information technology in colleges and universities cannot meet the needs of national talents, nor can they adapt to the needs of agricultural development in the new era, and there are deviations between campus teaching and practical application, and agricultural information technology graduate students do not have a comprehensive and accurate understanding and mastery of their specialties. At the same time, it is necessary to consider the logical relationship between various indicators and indicators at various levels. The selection of indicators should be consistent with the evaluation goals and reflect the conditions of the evaluated objects. Such an indicator system is more effective. The problems reflected by the training program are mainly the lack of practicality of degree public courses and professional courses, most of the courses in this area stay in books, the theory is greater than practice, theoretical courses are not updated on time to keep up with the times, course teaching is more boring, and the connection between agricultural scientific research and social production is interrupted, which cannot attract agricultural informatization graduate students to study in-depth, as shown in Figure 7.

The traditional model of agricultural informatization teaching has not kept pace with the development of science and technology, this solidified single teaching mode is somewhat backward and lacks innovation, the postgraduate students engaged in scientific research projects are mostly information technology-related rather than agriculture-related, lacking initiative and enthusiasm in learning agriculture-related knowledge, which is very unfavorable to understanding through the teaching content, and agricultural informatization professional teaching efficiency is also reduced. At the same time, agriculture and forestry colleges and universities do not fully use the rich information technology teaching resources in the new era, and the teaching methods are single and backward, and many new forms and new kinds of teaching methods are not applied to teaching, which is not conducive to the improvement of postgraduate learning and research ability. Therefore, the information technology teaching structure of agricultural and forestry colleges and universities should be reasonably adjusted, diversified teaching methods should be innovated, the overall teaching quality of agricultural and forestry teachers should be improved, and agricultural information technology postgraduates should be more actively integrated with agricultural knowledge and information technology to better retain high-tech talents for agricultural development.

The first thing that enterprises need to do to enhance the culture of the employer is to provide a good working environment and occupational safety. Formal means mainly adopt the form of text, voice, and image display, while informal means mainly adopt the form of description. The common method of realizing user portrait is like the pixels in imaging technology to describe the characteristics of people, which is the label in the portrait. A good working environment is based on harmonious employee relations and labor-management relations, and the harmony between employees and between employees and superiors can greatly improve efficiency and create benefits for the enterprise. Secondly, each employee must have a fair chance of promotion, which can stimulate employees' motivation and create a harmonious working environment. Finally, the employer can enhance the organizational culture of the unit by organizing activities to achieve the purpose of 


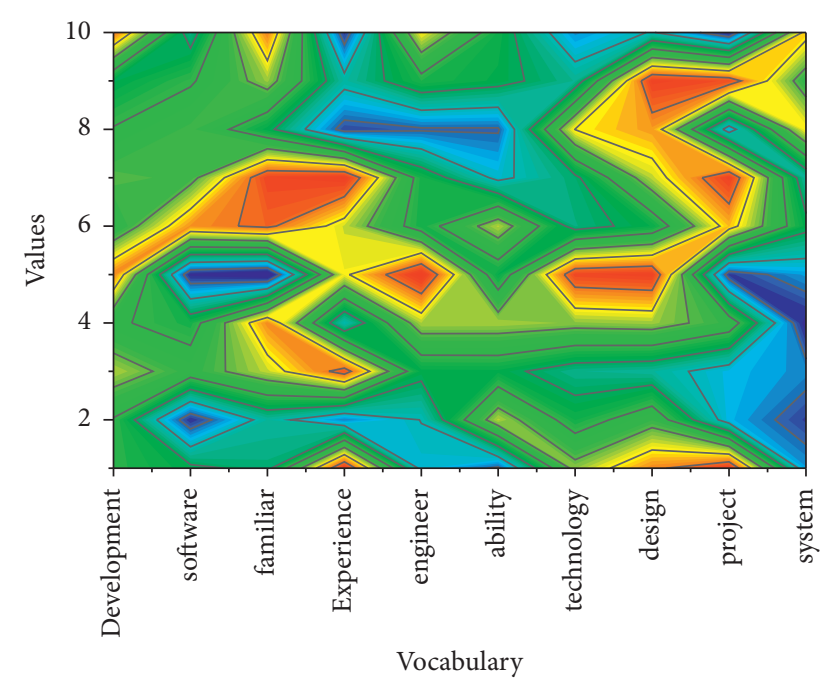

Figure 7: Results of factor determination.

strengthening the connection between employees and enhancing the cooperation between employees. Enhancing the culture of the employer not only enhances the cohesion among the employees of the enterprise, but also enhances the spiritual culture of the employer, thus enhancing the sense of access to the employment process of college students and improving the quality of employment.

\section{Conclusion}

The employment situation of college graduates in the big data environment is analyzed and studied. By taking a netend questionnaire survey and random interview with college graduates, it is found that more than 7 stone $\%$ of college graduates are confused about their future employment direction, and good employment analysis service has become a key work that cannot be ignored in the daily management of colleges and universities. Aiming at the problems of security that still need to be improved and low utilization rate of employment information in the employment information service platform of colleges and universities, it is proposed that colleges and universities can spare the help of big data technology to collect and summarize the employment information data of graduates, optimize the employment information service platform, timely understand and grasp the personal information of graduates and the job demands of employers, and then take targeted employment guidance and help for graduates, which improves the employment. The quality of service has been improved, and the employment quality of graduates has been enhanced. The employment information service platform can help employers understand all aspects of college graduates, such as education level, professional situation, and their quality. The campus recruitment network channel was set up specifically for graduates, which effectively promoted the employment of graduates. In the process of employment guidance in colleges and universities, employment management service systems such as online classes for employment guidance and professional career assessment have also been effectively used. Colleges and universities can carry out targeted employment guidance for graduates through a comprehensive understanding of all aspects of recruitment information released by employment units. Graduates can prepare for their employees according to their situation, to ensure that they can successfully take up jobs after graduation. The construction of big data employment information service platform can also provide strong support for the employment guidance work of colleges and universities. Employment guidance teachers can effectively carry out employment guidance work according to the information of graduates' professional characteristics, career regulation, and welfare policies of each region, collect a large amount of employment information with the help of big data technology, analyze its inner law, and provide graduates with targeted employment guidance and planning. Through the employment information service platform, instructors can fully understand the current social situation and job demands, reasonably adjust the relevant curriculum, innovate employment guidance concepts and methods, encourage students to boldly explore and practice, and enhance students' comprehensive literacy.

\section{Data Availability}

The data used to support the findings of this study are included within the article.

\section{Conflicts of Interest}

No conflicts of interest exist concerning this study.

\section{References}

[1] M. Attaran, J. Stark, and D. Stotler, "Opportunities and challenges for big data analytics in US higher education," Industry and Higher Education, vol. 32, no. 3, pp. 169-182, 2018.

[2] G. M. Slavich, S. Taylor, and R. W. Picard, "Stress measurement using speech: recent advancements, validation issues, and ethical and privacy considerations," Stress: The International Journal on the Biology of Stress, vol. 22, no. 4, pp. 408-413, 2019.

[3] N. R. Sanders, T. Boone, R. Ganeshan, and J. D. Wood, "Sustainable supply chains in the age of AI and digitization: research challenges and opportunities," Journal of Business Logistics, vol. 40, no. 3, pp. 229-240, 2019.

[4] T. Peele, V. Stoll, and A. Stella, "Teachers, researchers, and communities of practice: building a corpus to support graduate education," Journal of Basic Writing, vol. 37, no. 2, pp. 35-56, 2018.

[5] J. C. Burns, S. Chakraborty, and D. Saint Arnault, "Social media preference and condom use behaviors: an analysis of digital spaces with young african American males," Health Education \& Behavior, vol. 48, no. 2, pp. 190-198, 2021.

[6] E. Woyo, G. D. Rukanda, and Z. Nyamapanda, "ICT policy implementation in higher education institutions in Namibia: a survey of students' perceptions," Education and Information Technologies, vol. 25, no. 5, pp. 3705-3722, 2020.

[7] K. O'Halloran, “A posthumanist pedagogy using digital text analysis to enhance critical thinking in higher education," 
Digital Scholarship in the Humanities, vol. 35, no. 4, pp. $845-880,2020$.

[8] B. Shringarpure, "Africa and the digital savior complex," Journal of African Cultural Studies, vol. 32, no. 2, pp. 178-194, 2020.

[9] S. Arndt, G. Asher, J. Knox et al., "Between the blabbering noise of individuals or the silent dialogue of many: a collective response to "Postdigital science and education" (Jandrić et al. 2018)," Postdigital Science and Education, vol. 1, no. 2, pp. 446-474, 2019.

[10] T. Bennett, "Towards "Embedded Non-creative Work"? Administration, digitisation and the recorded music industry," International Journal of Cultural Policy, vol. 26, no. 2, pp. 223-238, 2020.

[11] N. Selwyn, L. Pangrazio, S. Nemorin, and C. Perrotta, "What might the school of 2030 be like? An exercise in social science fiction," Learning, Media and Technology, vol. 45, no. 1, pp. 90-106, 2020.

[12] E. Botezat, S. L. Fotea, M. Marici, and I. S. Fotea, "Fostering the mediating role of the feeling of belonging to an organization among Romanian members of generation Z," Studia Universitatis "Vasile Goldis" Arad - Economics Series, vol. 30, no. 4, pp. 69-91, 2020.

[13] F. L. Vallera and A. M. Bodzin, "Integrating STEM with AgLIT (agricultural literacy through innovative technology): the efficacy of a project-based curriculum for upper-primary students," International Journal of Science and Mathematics Education, vol. 18, no. 3, pp. 419-439, 2020.

[14] B. Ferdinandy, Á. M. Guerrero-Higueras, É. Verderber, F. J. Rodríguez-Lera, and Á. Miklósi, "Exploratory study of introducing HPC to non-ICT researchers: institutional strategy is possibly needed for widespread adaption," The Journal of Supercomputing, vol. 77, no. 5, pp. 4317-4331, 2021.

[15] D. M. Hilty, S. Chan, J. Torous, J. Luo, and R. J. Boland, "Mobile health, smartphone/device, and apps for psychiatry and medicine," Psychiatric Clinics of North America, vol. 42, no. 3, pp. 513-534, 2019.

[16] S. J. Andajani and A. Wijiastuti, "E-learning development for special education postgraduate students," International Journal of Emerging Technologies in Learning (iJET), vol. 15, no. 14, pp. 269-293, 2020.

[17] C. Hubert, F. Estrada, L. Campero, S. Des Deng, Y. Du, and L. Qi, "A web service composition approach based on planning graph and propositional logic," Journal of Organizational and End User Computing, vol. 31, no. 3, pp. 1-16, 2019.

[18] T.-H. Chou, "Exploring relationship quality of user's cloud service," Journal of Organizational and End User Computing, vol. 31, no. 3, pp. 17-36, 2019.

[19] M. Bevolo and F. Amati, "The potential role of aI in anticipating futures from a design process perspective: from the reflexive description of "design" to a discussion of influences by the inclusion of aI in the futures research process," World Futures Review, vol. 12, no. 2, pp. 198-218, 2020.

[20] A. Sarangapani, A. N. Simpson, J. Snelgrove, M. Higgins, R. Windrim, and A. Satkunaratnam, "Video-based teaching in patient and instrument selection for operative vaginal deliveries," Journal of Obstetrics and Gynaecology Canada, vol. 40, no. 9, pp. 1162-1169, 2018.

[21] N. Selwyn, S. Nemorin, and N. Johnson, "High-tech, hard work: an investigation of teachers' work in the digital age," Learning, Media and Technology, vol. 42, no. 4, pp. 390-405, 2017. 